\title{
Metástasis duodenogástrica por cáncer de mama: reporte de caso
}

\section{A Case Report of Duodenogastric Metastasis from Breast Cancer}

\author{
Daniel Andrés Santa Botero, ${ }^{1}$ Ángela Luna Meza, ${ }^{2}$ Camilo Castañeda Cardona, ${ }^{3}$ Diego Rosselli. ${ }^{4}$
}

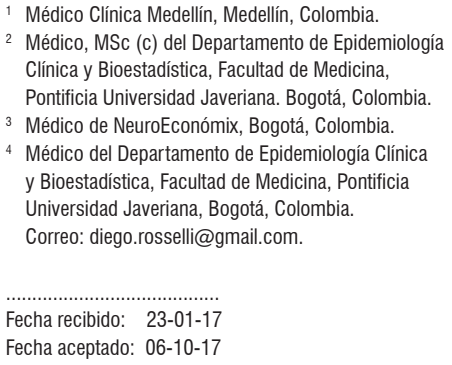

\begin{abstract}
Resumen
Se presenta el caso de una mujer de 82 años con cáncer de mama diagnosticado en 2011 tratado con cirugía y adyuvancia hormonal con tamoxifeno. En 2015 fue hospitalizada por obstrucción intestinal alta y se descartó compromiso tumoral. Sin embargo, se realizó videotoracoscopia por presencia de derrame pleural y se documentaron lesiones pleurales correspondientes a metástasis. Se inició terapia hormonal con adecuada respuesta. Un año después, se realizó gastroduodenoscopia de control en la que se evidenció edema y eritema bulboduodenal. La biopsia corroboró compromiso metastásico por cáncer de mama.
\end{abstract}

\section{Palabras clave}

Cáncer de mama; obstrucción intestinal; metástasis; fulvestrant; anastrozol.

\begin{abstract} duodenal bulb. The biopsy corroborated metastasis from breast cancer.

Keywords

Breast cancer; intestinal obstruction; metastasis; fulvestrant; anastrozole.
\end{abstract}

We present the case of an 82 year old woman who was diagnosed with breast cancer in 2011 and who underwent surgery and adjuvant treatment with tamoxifen. In 2015 she was hospitalized for an upper intestinal obstruction. Involvement of a tumor was ruled out, but video-assisted thoracoscopy showed the presence of pleural effusion and pleural lesions corresponding to metastases. Hormone therapy was initiated, and the patient responded adequately. One year later, a gastroduodenoscopy showed edema and erythema of the

\section{INTRODUCCIÓN}

El cáncer de mama es el cáncer más frecuentemente diagnosticado en el mundo y la primera causa de muerte por cáncer en mujeres. En Estados Unidos, se estima que se diagnostican 230000 casos nuevos cada año y corresponde a la causa de muerte de 40000 personas al año (1). En Colombia, se estima que se presentan más de 5000 casos nuevos de cáncer de mama cada año, con una tasa de incidencia entre 26,4 y 35,7/100 000 habitantes (2).
La expresión de receptores hormonales y HER2 permiten clasificar el cáncer de mama: el $80 \%$ de los casos tiene receptores hormonales positivos, el $23 \%$ tiene sobreexpresión de HER 2 y el $13 \%$ corresponde a cáncer de mama triple negativo (3). Los sitios más frecuentes de metástasis en cáncer de mama son el hueso, hígado y pulmones. La frecuencia de metástasis gástrica reportada es menor a $1 \%$ (4). Dada la incidencia creciente de cáncer de mama, así como la mayor supervivencia de las pacientes, es importante que todos los especialistas reconozcan las múltiples facetas de esta enfermedad. 


\section{DESCRIPCIÓN DE CASO}

A una mujer de 82 años, procedente de Medellín, se le diagnosticó cáncer de mama en 2011, estadio IIA (pT2pNOMOG2), receptores hormonales positivos y HER2 negativo. Se descartó la presencia de metástasis. Su único antecedente importante era taquicardia supraventricular, en manejo con bloqueante $\beta$ como tratamiento; se le realizó mastectomía radical modificada, adyuvante hormonal de mantenimiento con tamoxifeno y controles por oncología.

En septiembre de 2015 fue hospitalizada por obstrucción intestinal alta. Se le practicó entonces una endoscopia digestiva alta y, por estrechez duodenal, una colangiorresonancia. Se encontró dilatación de la cámara gástrica sin lesiones sugestivas de masa; sin embargo, se tomaron biopsias gastroduodenales que fueron negativas para malignidad. Durante la hospitalización presentó un síndrome broncoaspirativo que requirió manejo en la unidad de cuidados intensivos (UCI), con buena evolución clínica. La radiografía de tórax mostró derrame pleural, por lo que se llevó a videotoracoscopia, procedimiento en que se encontraron lesiones nodulares pleurales, cuya biopsia reportó compromiso por carcinoma de mama metastásico. El estudio de inmunohistoquímica mostró receptores hormonales positivos para estrógenos $100 \%$, progestágenos $10 \%$ y mamoglobina focal.

En diciembre de 2015, se valoró de forma extrainstitucional por oncología clínica y se inició terapia endocrina combinada con fulvestrant y anastrozol, con base en la evidencia presentada en el estudio SWOG 0226 (5), que mostró mejores tasas de respuesta y mayor tiempo de progresión que con anastrozol solo.

La paciente presentó buena tolerancia al tratamiento y ganancia de peso importante, por lo que, en mayo de 2016, al no tener razón suficiente para continuar con el bloqueo dual, se suspendió el anastrozol y se dejó monoterapia con fulvestrant, sin presentar efectos adversos. En junio de 2016, 6 meses después de haber iniciado el tratamiento, se realizó control imagenológico con tomografía toracoabdominal en la que se encontró respuesta completa.

En agosto de 2016, el gastroenterólogo solicitó una endoscopia de vías digestivas altas que evidenció edema y eritema bulboduodenal; la biopsia mostró presencia de células atípicas, el estudio de inmunohistoquímica fue positivo para los marcadores CK7, GATA 3, receptores de estrógenos positivos $(50 \%)$ y receptores de progestágenos negativos, corroborando compromiso por carcinoma de mama.

En este escenario, se considera que la paciente presentaba compromiso gastrointestinal por cáncer de mama, que corresponde con el síntoma inicial de la recaída en septiembre de 2015, a pesar de que en ese momento no se evidenciara compromiso tumoral en las biopsias gastroduodenales. La condición actual no indica progresión, ya que se trata de una paciente asintomática con estudios imagenológicos que así lo evidencian.

\section{DISCUSIÓN}

Existen 2 estudios sobre bloqueo hormonal dual con fulvestrant más inhibidor de aromatasa, con resultados contradictorios. El estudio fase II SWOG 0226 (5) mostró un beneficio en supervivencia libre de progresión de 15 meses con terapia combinada frente a 13,5 meses con monoterapia y una supervivencia global de 47 meses frente a 41,3 meses, respectivamente. Por el contrario, el estudio FACT (fulvestrant más anastrozol frente a anastrozol), fase II, no mostró beneficio clínico de la terapia combinada sobre la monoterapia (6).

Por otra parte, el estudio fase II FIRST, que comparó el tratamiento de primera línea para enfermedad metastásica con fulvestrant frente a anastrozol, mostró un beneficio con fulvestrant en supervivencia libre de progresión (23 frente a 13 meses) y supervivencia global ( 54 frente a 48 meses), y lo avaló como una terapia efectiva para el tratamiento de primera línea (7).

El cáncer de mama metastásico presenta una forma de diseminación y compromiso sistémico diferente según el subtipo histológico y molecular, luminal A, B o HER2 enrich; $y$ el tracto gastrointestinal tiene menor probabilidad de presentar metástasis. Ambroggi y colaboradores describieron a 5 pacientes con compromiso gastrointestinal por cáncer de mama y realizaron una revisión sistemática de la literatura que comprendió el período de 1943 a 2012, en la que se encontraron reportados tan solo 265 casos de este tipo (8). El manejo del cáncer de mama metastásico no cambia según la región comprometida y la mediana de la supervivencia en estos casos es muy variable, entre 3 y 10 años, dependiendo del control molecular y de la sensibilidad propia de cada paciente.

El caso presentado ilustra el compromiso duodenal y gástrico por cáncer de mama luminal $\mathrm{A}$, lo cual plantea un diagnóstico diferencial a tener en cuenta en las pacientes con enfermedad de mama metastásica y sintomatología gastrointestinal. A pesar de que se trata de una única paciente, es probable que la terapia con fulvestrant y anastrozol sea la responsable de su buena respuesta clínica.

\section{Conflicto de interés}

Este trabajo no recibió financiación externa; los autores no declaran ningún conflicto de interés. 


\section{REFERENCIAS}

1. Siegel RL, Miller KD, Jemal A. Cancer statistics, 2015. CA Cancer J Clin. 2015;65(1):5-29. https://doi.org/10.3322/ caac. 21254 https://doi.org/10.3322/caac.21314

2. Alba LH, Alba M, Ortiz D, et al. Análisis de los registros individuales de prestación de servicios de salud (RIPS) en cáncer en Colombia. Medicina (Bogotá). 2016;38(3):223-31.

3. Lobbezoo DJ, van Kampen RJ, Voogd AC, et al. Prognosis of metastatic breast cancer subtypes: the hormone receptor/ HER2-positive subtype is associated with the most favorable outcome. Breast Cancer Res Treat. 2013;141(3):507-14. https://doi.org/10.1007/s10549-013-2711-y

4. Lambert LA. 3-27 Breast cancer: Presentation and intervention in women with gastrointestinal metastasis and carcinomatosis. Breast Dis. 2006;17(3):250-1. https://doi. org/10.1016/S1043-321X(06)80509-6

5. Mehta RS, Barlow WE, Albain KS, et al. Combination anastrozole and fulvestrant in metastatic breast cancer. N Engl
J Med. 2012;367(5):435-44. https://doi.org/10.1056/ NEJMoa 1201622

6. Bergh J, Jönsson PE, LidbrinkEK, et al. FACT: an open-label randomized phase III study of fulvestrant and anastrozole in combination compared with anastrozole alone as first-line therapy for patients with receptor-positive postmenopausal breast cancer. J Clin Oncol. 2012;30(16):1919-25. https:// doi.org/10.1200/JCO.2011.38.1095

7. Robertson JF, Lindemann JP, Llombart-Cussac A, et al. Fulvestrant $500 \mathrm{mg}$ versus anastrozole $1 \mathrm{mg}$ for the firstline treatment of advanced breast cancer: follow-up analysis from the randomized 'FIRST' study. Breast Cancer Res Treat. 2012;136(2):503-11. https://doi.org/10.1007/ s10549-012-2192-4

8. Ambroggi M, Stroppa EM, Mordenti P, et al. Metastatic breast cancer to the gastrointestinal tract: report of five cases and review of the literature. Int J Breast Cancer. 2012;2012:439023. 